\title{
THE GEOMETRY OF FINSLER SPACES
}

\author{
HERBERT BUSEMANN
}

The term "Finsler space" evokes in most mathematicians the picture of an impenetrable forest whose entire vegetation consists of tensors. The purpose of the present lecture is to show that the association of tensors (or differential forms) with Finsler spaces is due to an historical accident, and that, at least at the present time, the fruitful and relevant problems lie in a different direction.

Finsler spaces were discovered by Riemann in his lecture [1]:1 Über die Hypothesen, welche der Geometrie zu Grunde liegen (1854). The goal which Riemann set for himself was the definition and discussion of the most general finite-dimensional space in which every curve has a length derived from an infinitesimal length or line element. In modern terminology Riemann's approach is this: Let a differentiable manifold $M$ of a certain class be given. In any local coordinate system $\left(x_{1}, \cdots, x_{n}\right)=(x)$ a length $F(x, d x)$ must be assigned to a given line element $(x, d x)=\left(x_{1}, \cdots, x_{n} ; d x, \cdots, d x_{n}\right)$ with origin $x$. If $x(t)$ is a (smooth) curve in $M$ then $\int F(x, \dot{x}) d t$ is its length.

In order to insure that the length of a curve is positive and independent of the sense in which the curve is traversed, Riemann requires $F(x, d x)>0$ for $d x \neq 0$ and $F(x, d x)=F(x,-d x)$.

Next Riemann assumes $[1$, p. 277] that the length of the line element remains unchanged except for terms of second order, if all points undergo the same infinitesimal change. This amounts to the condition $F(x, k d x)=k F(z, d x)$ for $k>0$. Nowadays we rather justify this condition by requiring that a change of the parametrization of the curve does not change its length.

Riemann then turns immediately to the special case where $F(x, d x)$ $=\left[\sum g_{i k}(x) d x_{i} d x_{k}\right]^{1 / 2}$, that is, to those spaces which are now called Riemann spaces. The general case is passed over with the following remarks: the next simplest case would comprise the manifolds, in which the line element can be expressed as the fourth root of a biquadratic differential form. The investigation of these more general types would not require any essentially different principles, but it would be time consuming and contribute comparatively little new to the theory of space (verhältnismässig auf die Lehre vom Raume wenig

An address delivered before the Los Angeles Meeting of the Society on November 27, 1948, by invitation of the Committee to Select Hour Speakers for Far Western Sectional Meetings; received by the editors December 8, 1948.

${ }^{1}$ Numbers in brackets refer to the references cited at the end of the paper. 
neues Licht werfen), because the results cannot be interpreted geometrically (see [1, p. 278]).

Here is one of the few instances where Riemann's feeling was wrong. ${ }^{2}$ Nevertheless the passage had a great influence: the general case was for a long time entirely neglected, and when it was taken up the principles of Riemannian geometry were applied. The results thus obtained are not different enough to enrich geometry materially, moreover they frequently do not lend themselves to a naîve geometric interpretation.

The following is a brief sketch of the history. The integral $\int F(x, \dot{x}) d t$ is the subject of the Weierstrass theory in the calculus of variations. Therefore it is not surprising that the first contributions are due to workers in this field. The pioneers are Bliss $[2,3]$ and his students (in particular Underhill [4]) in this country and Landsberg [5] in Germany. To develop a geometry it is necessary to have extremals or geodesics whose subarcs furnish locally unique shortest connections. The Legendre or Weierstrass conditions of the calculus of variations suggest to require that $F(x, d x)$ is for fixed $x$ a convex function of $d x$ :

$$
F(x, d x)+F(x, \delta x) \geqq F(x, d x+\delta x)
$$

or, which is the same, that $F(x, y)=1$ is a convex surface in $y$-space. (1) will be assumed henceforth. ${ }^{8}$

Bliss' approach is as follows: the homogeneity of $F(x, d x)$ in $d x$ permits to write $F(x, d x)$ in the form

$$
F(x, d x)=\left[\sum g_{i k}(x, d x) d x_{i} d x_{k}\right]^{1 / 2}
$$

where the $g_{i k}(x, d x)$ are homogeneous of degree 0 in $d x$.

If a field of curves is distinguished and $\delta(x)$ is tangent to the field at $x$, then $\sum g_{i k}(x, \delta(x)) d x_{i} d x_{k}$ is the line element of a Riemann space. The methods of Riemannian geometry are applicable, but the

2 It is probable that he did not give the matter much thought. The lecture [1] was to give him the privilege to teach at the University of Göttingen. The candidate had to propose three topics, and the faculty selected one of them. Riemann had put the present topic last. He did not expect to work it out and was pressed for time, when it was selected. This is evidenced by the following passage from a letter to his brother Wilhelm, dated December 8, 1853: . . . und musste dabei drei Themata zur Probevorlesung vorschlagen, von denen dann die Fakultät eins wählt. Die beiden ersten hatte ich fertig und hoffte dass man eins davon nehmen wirde; Gauss aber hatte das dritte gewählt, so bin ich wieder etwas in der Klemme, da ich dies noch ausarbeiten muss.

${ }^{3}$ On the other hand, many results hold without Riemann's condition $F(x, d x)$ $=F(x,-d x)$. The present lecture supposes, however, that this relation holds. In the sequel it will be convenient to use the term line element both for $d s=F(x, d x)$ and for $d s^{2}$. 
results depend on the choice of the field. For instance, the Gauss-Bonnet Theorem has been investigated by Bliss [3] from this point of view.

Finsler was the first who studied the general spaces systematically in his thesis [6] written under Carathéodory's guidance. His main idea is this: if a curve is given, then a field which contains the curve defines a Riemann metric as above. Those results which are independent of the choice of the field are the real geometric properties of the curve. In this way Finsler succeeded in developing a theory of curves, and also the foundations for a theory of surfaces. This is the reason why the name Finsler space was generally accepted for the general class of spaces first defined by Riemann.

Then a new line of thought developed in the geometric school at Prague with Berwald, Funk, and Winternitz as principle representatives. ${ }^{4}$ A Finsler space is not considered as a point space but primarily as a set of line elements in which a Riemannian metric is associated with each line element. The main emphasis in this theory lies on the definitions and properties of parallelism and similar questions. The whole development culminated in Cartan's monograph [8], which is considered to have given the theory of Finsler spaces its final form (endgültige Gestalt, see [7, p. 1]).

Under the restriction to use nothing but Riemannian methods it may be true that the theory has reached its limits. However, as soon as this restriction is dropped it appears that the surface has hardly been scratched.

A first, but not the most interesting or decisive, step beyond the mentioned results consists in extending the results of Riemannian geometry rather than its methods.

As an example consider the following statement: In a Riemann space $R$ with nonpositive curvature there is exactly one geodesic arc connecting two points within a given homotopy class. A real geometer will be convinced that such a nice geometric property must have a simple geometric core which has nothing to do with the Riemannian character of the metric. Guided by this conviction he soon finds that a Riemann space has nonpositive curvature if and only if in small geodesic triangles $a b c$ the (shortest) geodesic connection of the mid points $b^{\prime}, c^{\prime}$ of the sides $a b$ and $a c$ is at most half as long as the third side $b c$ :

$$
2 b^{\prime} c^{\prime} \leqq b c .
$$

This inequality makes sense in any Finsler space. If it is assumed

${ }^{4}$ For the rather voluminous litera ture see the excellent report [7] of Berwald. 
(locally) then it turns out, see [9], that not only the mentioned theorem, but the whole beautiful theory of spaces with nonpositive curvature holds as it was developed by Hadamard [10] and Cartan [11, Note III]. The proofs are, however, quite different, for instance properties of convex functions replace the Gauss-Bonnet Theorem. It should also be noted that (3) does not pressuppose differentiability properties, and that, in fact, the theory holds without them.

This is only one example among many. Very frequently, when the conclusion of a theorem on Riemann spaces is a simple geometric fact, the hypothesis can be freed of its Riemannian character and the theorem then carries over to Finsler spaces.

But investigations of this type yield nothing but direct analogs to Riemannian results and are not fundamentally new. Poincaré said somewhere ${ }^{5}$ on the subject of generalizations: On ne fait pas un grand voyage pour ne trouver que ce qu'on a chez soi. In the case of Finsler spaces a long trip will pay, because they are really fundamentally different from Riemann spaces. Just now we have reached a stage where we begin to understand the problems.

Let us start with volume and area. The problem how to define these concepts was among the first which attracted the attention in Finsler spaces. The approach was as follows:

The volume element of a Riemann space is $\left|g_{i k}(x)\right|^{1 / 2} d x_{1} \cdots d x_{n}$, where $\left|g_{i k}(x)\right|$ is the determinant of the $g_{i k}(x)$. We wish to find an expression in the derivatives of $F(x, d x)$ which reduces to $\left|g_{i k}(x)\right|^{1 / 2}$ in the Riemannian case. This turns out to be possible in infinitely many ways. Consequently there seemed to be many possible definitions of volume, and the problem was considered as fruitless.

Is this conclusion justified? An unprejudiced attack on the problem would doubtless begin with the question: Why are the definitions of volume and area unique in the Riemannian case? The answer is simple, but rests on two fundamental principles which are usually not formulated explicitly. Let $G$ be a bounded domain in a Riemann space $R$ whose volume is to be determined. Guided by the procedure employed in integration, we divide $G$ into small domains $G_{\nu}$. In each $G_{\nu}$ select a point $x^{\nu}$ and replace $g_{i k}(x)$ in $G_{\nu}$ by $g_{i k}\left(x^{\nu}\right)$. With this metric $G_{\nu}$ is euclidean. We know what volume in a euclidean space means. Its value for $G_{\nu}$ is $\left|g_{i k}\left(x^{\nu}\right)\right|^{1 / 2} \iint_{\sigma_{\nu}} d x_{1} \cdots d x_{n}$. By the usual limit process of integration we find $\iint_{G}\left|g_{i k}(x)\right|^{1 / 2} d x_{1} \ldots d x_{n}$ as Riemannian volume of $G$. The principle which underlies this procedure is clearly this: A Riemannian metric is locally euclidean. At every point

5 The author forgot where he saw this remark and was unable to locate it, but would be grateful for a reference. 
the volume element of the space must coincide with the volume element of the local geometry.

Turning to area we observe: If $x_{i}\left(u_{1}, \cdots, u_{k}\right)=x(u)$ is a $k$-dimensional submanifold $M$ of $R$, then the metric $R$ induces on $M$ the metric

$$
d \sigma^{2}=\sum_{i, k, j, h} g_{i k}(x(u)) \frac{\partial x_{i}}{\partial u_{j}} \frac{\partial x_{k}}{\partial u_{h}} d u_{j} d u_{h}=\sum_{j, h} \gamma_{j h}(u) d u_{j} d u_{h}
$$

With this metric $M$ becomes a $k$-dimensional Riemann space. Its volume must be the area of $M$ as manifold in $R$. This statement is based on the principle that area must be intrinsic, that is, depend only on the $\gamma_{j h}(u)$.

We now try to treat volume and area in a Finsler space $R$ with line element $F(x, d x)$ by using the same principles. We divide a given domain $G$ in $R$ into small domains $G_{\nu}$. In $G_{\nu}$ we select a point $x^{\nu}$. In $G_{\nu}$ we replace $F(x, d x)$ by $F\left(x^{\nu}, d x\right)$. But there it seems to end, because the space with $F\left(x^{\nu}, d x\right)=F(d x)$ as line elements is not euclidean, but is a Minkowskian (or finite-dimensional Banach) space $S$.

If $d x$ is replaced by $x$ the distance of two points $x, y$ in $S$ is given by

$$
F(y-x)=|y-x| \cdot F\left(\frac{y-x}{|y-x|}\right)=|y-x| \cdot F(u)
$$

where $u$ is a unit vector in the direction of the vector $y-x$. Therefore the Minkowski distance originates from the euclidean distance $y-x$ by multiplying it with a factor which depends only on the direction of the segment from $x$ to $y$. The triangle inequality holds because of (1). The euclidean segments are also shortest connections for the Minkowski metric, and the only ones when the unit sphere $F(x)=1$ is strictly convex. ${ }^{6}$ The same Minkowski space can be derived from different euclidean spaces $(x)$ in the form (4). All these spaces are related by nondegenerated affine transformations and are called associated to the Minkowski space, compare $[13, \S 2]$.

Obviously the Minkowski distance (4) is invariant under the translations $x^{\prime}=x+a$. Since these operate in a simply transitive way on the space, the theory of Haar measure shows that $u p$ to a constant factor at most one measure exists which is invariant under the translations. One such measure is the Lebesgue measure $|M|_{n}^{L}$ of a set $M$ in the euclidean space $(x)$ with distance $|y-x|$. This measure itself cannot be used because it depends on the choice of the associated space. Thus the question reduces to determining $\lambda$ such that

- For these statements compare [12, § II.1]. 


$$
|M|_{n}=\lambda|M|_{n}^{L} \text { is an acceptable measure in } S .
$$

Several possibilities suggest themselves: the distance $F(y-x)$ or one-dimensional measure can be interpreted as follows: The Minkowskian unit sphere $V: F(x, y)=1$ is convex and has $O$ as center. For given points $x, y$ let $z_{1} z_{2}$ be the diameter of $V$ parallel to the line $x y$ or the intersection $\alpha$ of the line parallel to $x y$ through $O$ with the solid sphere $W: F(x) \leqq 1$. Then $F\left(z_{2}-z_{1}\right)=2$ and since $(y-x) /|y-x|$ $=\left(z_{2}-z_{1}\right) /\left|z_{2}-z_{1}\right|$

$$
\begin{aligned}
F(y-x) & =\frac{|y-x|}{\left|z_{2}-z_{1}\right|} F\left(z_{2}-z_{1}\right)=\frac{2}{\left|z_{2}-z_{1}\right|}|y-x| \\
& =\frac{2}{|\alpha|_{1}^{L}}|y-x| .
\end{aligned}
$$

Observing that 2 is the volume of the one-dimensional euclidean unit sphere and keeping the principle of intrinsiqueness in mind, we are led to the following choice of $\lambda$ : Let $N$ be a set in a $k$-dimensional, $0<k \leqq n$, linear space $L$. Let a $k$-dimensional space parallel to $L$ through $O$ intersect $W$ in the set $\alpha$ and define in analogy with (5)

$$
N_{k}=\frac{\Omega_{k}}{|\alpha|_{k}^{L}} \cdot|N|_{k}^{L}, \quad \Omega_{k}=\pi^{n / 2} \Gamma^{-1}\left(\frac{n}{2}+1\right) \cdot{ }^{7}
$$

On the other hand Hausdorff measure could be used. In fact there are two Hausdorff measures: in one definition the set $N$ is covered by spheres of diameter less than $\epsilon$, and in the other by arbitrary sets of diameter less than $\epsilon$. The equality of these measures in the euclidean case depends on the fact that the sphere maximizes the volume among all sets with a given diameter (see, for instance, $[13$, p. 239]).

Since the Minkowski spheres also maximize the volume among all sets of a given Minkowski diameter [13, pp. 243-246], the two $k$ dimensional Hausdorff measures are equal for the above set $N$. What is more, they also yield the value $\Omega_{k} /|\alpha|_{\boldsymbol{k}}^{L}$ for $\lambda$. There are other cogent reasons $[13$, pp. 242,243$]$ which lead to the same choice of $\lambda$. Consequently, measure in Minkowski spaces is just as uniquely determined and therefore as interesting as in euclidean spaces.

The principle that the volume element of a space at a point must coincide with the volume element of the local space then determines volume in Finsler spaces. Area is defined by the same procedure as in

${ }^{7}$ So that $\Omega_{k}$ is the volume of the $k$-dimensional euclidean unit sphere. It should be shown that $|N|_{k}$ is independent of the associated metric, but this is contained in what follows. 
Riemann spaces using the principle that area is intrinsic.

Hence, there is just as little choice for the definition of volume and area in Finsler spaces as there is in Riemann space. How could this definition then have been missed by others ?8 The volume element corresponding to (6) is an integro-differential expression, and the preconceived idea that it must be a differential expression barred the way.

In order to discover what volume and area in Finsler spaces are, Minkowski spaces had to be investigated, just as euclidean geometry furnished the clue for Riemann spaces. But euclidean geometry does more for Riemann spaces, not only volume and area, but all the basic concepts of Riemannian geometry like angle, curvature of curves and surfaces are dictated by the corresponding euclidean concepts. Cartan's approach to Riemannian geometry in [11] is based on this idea. Clearly the step from no geometry to euclidean geometry is incomparably much wider than that from euclidean geometry to Riemannian geometry.

The volume problem makes it more than probable that an analogous situation exists for Finsler spaces. Therefore the study of Minkowskian geometry ought to be the first and main step, the passage from there to general Finsler spaces will be the second and simpler step.

What has been done in Minkowskian geometry, what are the difficulties and problems, and which tools will be necessary? Little has been done, but the field is quite accessible. The main difficulty comes from our long euclidean tradition, which makes it hard (at least for the author) to get a feeling for the subject and to conjecture the right theorems.

The type of problem which faces us is clear: A Minkowskian geometry admits in general only the translations as motions and not the rotations. Since the group of motions is narrower we expect more invariants. By passing from euclidean to projective geometry, ellipses, parabolas, and hyperbolas become indiscernible. The present case presents the much more difficult converse problem, to discern objects which have always been considered as identical.

An example will make this clearer. A sphere $V(p, \rho)$ in the euclidean or Minkowskian space is defined as the locus of those points $x$ whose distance $p x$ from $p$ equals $\rho$. Let $W(p, \rho)$ denote the set of points $x$ with $p x \leqq \rho$. In the euclidean case the sphere has the following other

8 Actually Choquet gave this definition in [14], but only as one possibility among several, so that the uniqueness properties of this area were not noted, nor are there any theorems on this area. I also heard that $\mathrm{C}$. Loewner proposed in conversations the use of this area. 
properties:

(1) $W(p, \rho)$ maximizes the volume among all sets of diameter $2 \rho$.

(2) $V(p, \rho)$ solves the isoperimetric problem.

(3) Spheres lead to the area $A(s)$ of a convex or sufficiently smooth non-convex simple closed surface $S$ bounding a set $K$ through the relation

$$
A(s)=\lim _{\rho}^{-1}\left(\left|K_{\rho}\right|_{n}^{L}-|K|_{n}^{L}\right)
$$

where $K_{\rho}=\cup_{x \in K} W(x, \rho)$.

(4) $V(p, \rho)$ is envelope of planes normal to the rays with origin $p$.

It was already mentioned that Minkowski spheres have property (1) which is decisive for measure theory. But a Minkowski sphere does, in general, not solve the isoperimetric problem for the area defined above. The solutions are found by applying the Brunn-Minkowski theorem, see [15]. They are homothetic to each other. It is convenient to single out one of them $T=T(o, 1)$, by the requirement that it has $o$ as center and that its Minkowski area is $n$ times its Minkowski volume. ${ }^{9}$

The relation (7) does not hold for the Minkowski area $A_{m}(s)$ of $S$ and the Minkowski spheres $W(p, \rho)$. However, if $T(p, \rho)$ denotes the surface obtained from $T(o, 1)$ by the dilation $x^{\prime}=\rho x$ followed by the translation $x^{\prime \prime}=x^{\prime}+p$ and $W^{*}(p, \rho)$ is the closed set bounded by $T(p, \rho)$, then (7) holds in the form

$$
A_{m}(S)=\lim \rho^{-1}\left(\left|K_{\rho}^{*}\right|_{n}-|K|_{n}\right)
$$

where $K_{\rho}^{*}=U_{x \in K} W^{*}(x, \rho)$, see [15].

Thus $T(p, \rho)$ has two properties of the euclidean sphere.

Property (4) proves particularly illuminating. A line $L$ in euclidean or in Minkowskian geometry is normal to a set $M$ at a point $f \in M$ if $L$ contains $f$ and every point $x$ of $L$ has $f$ as foot on $M$, that is, if $x y \geqq x f$ for $x \in L$ and $y \in M$. In euclidean geometry normality of a line to a line $L^{\prime}$ or a hyperplane $H$ at $f$ implies normality at $f$ of $L^{\prime}$ to $L$ or normality to $L$ of every line in $H$ through $f$. It is well known from the calculus of variations (where the word transversality is used) and from the theory of Banach spaces that this is not so in Minkowski spaces.

If $H$ is a supporting plane of the Minkowski sphere $V(p, \rho)$ at a point $f$, then $L=p f$ is normal to $H$ and to every line in $H$ through $f$.

\footnotetext{
- The area of the euclidean unit sphere is $n \Omega_{n}$. The corresponding relation is not true for the Minkowskian $V(0,1)$ and $W(0,1)$.
} 
Therefore a Minkowski sphere is still envelope of planes to which the radii are normal.

Is it also the envelope of planes normal to the radii? Normality of a plane to a line is as yet undefined, but it is easy to see how it can be defined. That $L$ is normal to $H$ may be expressed as follows: if $H_{1}, H_{2}$ are any two planes parallel to $H$, then the segment intercepted by $H_{1}, H_{2}$ on $L$ is not longer than a segment intercepted on any other line $L^{*}$ or also: the one-dimensional measure of a set on a straight line $L^{*}$ not parallel to $H$ is at least as large as the measure of its projection parallel to $H$ on $L$.

It is then natural to call the hyperplane $H$ normal to the line $L$ if the $(n-1)$-dimensional Minkowski measure (6) of a set in a hyperplane $H^{*}$ not parallel to $L$ is at least as large as the measure of its projection parallel to $L$ on $H$.

The existence of a hyperplane normal to a given line $L$ is trivial and not very interesting. But the converse question, whether for a given hyperplane $H$ a line $L$ exists to which $H$ is normal, is very interesting, since a positive answer implies among other things that hyperplanes are minimal (in the sense of area minimizing) surfaces. ${ }^{10}$

The question turns out to be equivalent to the following problem on convex bodies: Let $W$ be a convex body with center $o$. For any hyperplane $H$ through $o$ lay off on the normal to $H$ at $o$ (in both directions) the $(n-1)$-dimensional volume $|H \cap W|_{n-1}^{L}$ of $H \cap W$. Is the locus $Y$ of the points thus obtained a convex surface?

The answer is positive, but not trivial, unless the proof in [16] can be considerably simplified. This whole discussion shows, what should have been a truism from the beginning, that the theory of convex bodies is one of the essential tools for Finsler spaces.

Returning to property (4) we ask: what are the envelopes of the planes normal to the rays issuing from a fixed point $p$ ? They are found to be the solutions $T(p, \rho)$ of the isoperimetric problem. The deeper reason for this surprising fact is that for $W=W(0,1)$ the above constructed surface $Y$ is related to $T(0,1)$ by a polar reciprocity with respect to a euclidean sphere $\sum x_{i}^{2}=r^{2}$ with a suitable radius $r>0$.

Thus property (4) divides into two properties, the spheres $V(p, \rho)$ have one of them and the solution $T(p, \rho)$ of the isoperimetric problem the other.

If the definition and four properties of euclidean spheres had all led to different Minkowskian surfaces, the theory would be too disorganized to be interesting. Had they all led to the Minkowski sphere,

${ }^{10}$ These and some of the following statements will be proved in The foundations of Minkowskian geometry, which is going to appear in the Comment. Math. Helv. 
the above quoted aphorism of Poincare would apply. The fact that just two surfaces emerged seems to promise an interesting future.

The properties of Minkowskian geometry hitherto discussed are of the most primitive nature. This reflects the actual state of the theory, with one exception: the differential geometric results connected with the sphere as carrier of the spherical image have been extended to Minkowski spaces in so-called relative differential geometry. Since this branch is almost unknown in America, a digression on its origin and nature may be useful. Minkowski [17, \$24] observed that (7) yields

$$
A(S)=n V_{1}(K, W(0,1))
$$

where $V_{i}\left(L_{1}, L_{2}\right)$ denotes generally the mixed volume of $n-i$ times the convex body $L_{1}$ and $i$ times the convex body $L_{2}$ (compare [19, $\$ \$ 29$ and 30$]$ ). Guided by (9) he defined $[17, \$ 27]$ for an arbitrary convex body $L$

$$
A_{L}(S)=n V_{1}(K, L)
$$

as area of $S$ relative to $L$. Minkowski showed moreover $[18, \S 4]$ that the mixed volumes $V_{i}(K, W(0,1))$ are in $E^{3}$ closely related to the elementary symmetric functions of the principle radii of curvature of $S$, and that similar statements apply to $V_{i}(K, L)$. This theory was continued by others, in particular extended to $E^{n}$ (see $[19, \S 38]$ ). Thus a differential geometry relative to $L$ was developed. ${ }^{11}$

It was not recognized that its results contribute to Minkowskian geometry because they do not yield theorems on the Minkowski geometry $\mathcal{X}$ with $V(o, 1)$ as unit sphere if applied to $W(o, 1)$ as $L$. However (8) shows that

$$
A_{m}(S)=n V_{1}(K, T) .
$$

Therefore the differential geometry relative to $T$ belongs to $\mathcal{X}$.

A typical result, contained in Duschek [20] and derived under the usual unspecified differentiability assumptions of differential geometry, is the following:

Map a given surface $S$ in a three-dimensional Minkowski space on $T$ by associating points $x$ of $S$ and $x^{\prime}$ of $T$ where the tangent planes are parallel. At any point $x$ of $S$ there are exactly two directions $d_{1}, d_{2}$ which are parallel to the corresponding directions $\delta_{1}, \delta_{2}$ at $x^{\prime}$, so that, corresponding to the formula of Olindes Rodrigues in ordinary differential geometry, $d_{i} x=R_{i} \delta_{i} x^{\prime}$. The $R_{i}$ do not depend on the associated euclidean metric and have therefore a Minkowskian meaning.

${ }^{11}$ A complete list of the literature until 1934 is found in $[19$, p. 65]. 
Duschek shows that several of the usual interpretations of $R_{i}$ can be extended to the present case and proves moreover: $S$ is a minimal surface, that is, the first variation of the Minkowski area of $S$ vanishes, if and only if $R_{1}^{-1}+R_{2}^{-1} \equiv 0$.

Reassuring as these results are, they bypass technically simpler, but much more basic, questions which ought to be answered first. The first theorem of ordinary differential geometry is the statement that the curvature determines a plane curve. More explicitly: if $p(s), p^{\prime}(s), o \leqq s \leqq \alpha$, are arcs in $E^{2}$ with the arclength $s$ as parameter and equal curvature for equal $s$, then a motion of $E^{2}$ exists which carries $p(s)$ into $p^{\prime}(s)$ for $o \leqq s \leqq \alpha$. In $E^{2}$ this is equivalent to

$$
p\left(s_{1}\right) p\left(s_{2}\right)=p^{\prime}\left(s_{1}\right) p^{\prime}\left(s_{2}\right) \quad \text { for } o \leqq s_{i} \leqq \alpha .
$$

In a Minkowski plane the relation (10) may hold for arcs for which no motion of the plane exists which carries $p(s)$ into $p^{\prime}(s)$.

The first problem of Minkowskian differential geometry is therefore, clearly, to discuss the meaning of the statement that an arc is determined, and then to find curvature functions which determine the arc in some sense, for instance (10). But the problem has never been treated. ${ }^{12}$

This confirms that in spite of all the work on Finsler spaces we are now at a stage which corresponds to the very beginning in the development of ordinary differential geometry. Therefore the mathematician who likes special problems has the field. After sufficiently many special results have been accumulated someone will create the appropriate tools. At the present time it is difficult to guess what they will be beyond a vague feeling that some theory of integrodifferential invariants will be essential.

\section{REFERENCES}

1. B. Riemann, Über die Hypothesen, welche der Geometrie zu Grunde liegen, Mathematische Werke, Leipzig, 1892, pp. 272-287.

2. G. A. Bliss, $A$ generalization of the notion of angles, Trans. Amer. Math. Soc. vol. 7 (1906) pp. 184-196.

3. - Generalizations of geodesic curvature and a theorem of Gauss concerning geodesic triangles, Amer. J. Math. vol. 37 (1915) pp. 1-18.

4. A. L. Underhill, Invariants of the function $F\left(x, y, x^{\prime}, y^{\prime}\right)$ in the calculus of variations, Trans. Amer. Math. Soc. vol. 9 (1908) pp. 316-338.

5. G. Landsberg, Ueber die Krilmmung in der Variationsrechnung, Math. Ann. vol. 65 (1908) pp. 313-349.

6. P. Finsler, Ueber Kurven und Flächen in allgemeinen Räumen, Dissertation,

12 While this paper was in print the author has discussed the problem in the paper quoted in footnote 10 with the result, that two curvatures, instead of one, determine a curve in a sense which is stronger than (10) but a little weaker than mobility of $p(s)$ into $p^{\prime}(s)$. 
Göttingen, 1918.

7. L. Berwald, Ueber Finslersche und verwandte Räume, Comptes Rendus du deuxième congrès des mathematiciens des pays slaves, Prague, 1935, pp. 1-16.

8. E. Cartan, Les espaces de Finsler, Exposées de Géométrie, vol. 2, Paris, 1934.

9. H. Busemann, Spaces with non-positive curvature, Acta Math. vol. 80 (1948) pp. 259-310.

10. J. Hadamard, Les surfaces a courbures opposées et leur lignes géodésiques, J. Math. Pures Appl. (5) vol. 4 (1898) pp. 27-730.

11. E. Cartan, Leçons sur la géométrie des espaces de Riemann, Paris, 1928.

12. H. Busemann, Metric methods in Finsler spaces and in the foundations of geometry, Annals of Mathematics Studies, no. 8, Princeton, 1942.

13. - Intrinsic area, Ann. of Math. vol. 48 (1947) pp. 234-267.

14. G. Bouligand and G. Choquet, Problèmes liés à des métriques variationnelles, C. R. Acad. Sci. Paris vol. 218 (1944) pp. 696-698.

15. H. Busemann, The isoperimetric problem for Minkowski area, Amer. J. Math. vol. 71 (1949) pp. 743-762.

16. - A theorem on convex bodies of the Brunn-Minkowski type, Proc. Nat. Acad. Sci. U.S.A. vol. 35 (1949) pp. 27-31.

17. H. Minkowski, Theorie der konvexen Körper, Gesammelte Abhandlungen, vol. 2, Leipzig, 1911, pp. 131-229.

18. —, Volumen und Oberflache, ibid. pp. 230-276.

19. T. Bonnesen and W. Fenchel, Theorie der konvexen Körper, Berlin, 1934.

20. A. Duschek, Ueber relative Flächentheorie, Sitzungsberichte der Akademie der Wissenschaften, Wien vol. 135 (1926) Abteilung IIa, pp. 1-8.

The University of Southern California 